\title{
STRUCTURAL AND OPTICAL CHARACTERIZATION OF
}

\section{UNDOPED AND COPPER DOPED CdS NANOPARTICLES}

\section{T. Nanhoriya ${ }^{1}$, K. Vishwakarma ${ }^{1}$, K. Kushwah ${ }^{2}$, M. Ramraikhiani $^{3}$}

${ }^{1}$ Dept. of Nanotechnology, Gyan Ganga College of Technology, Jabalpur, 2Dept. of App. Physics, Jabalpur Engg. College, Jabalpur, M.P. India

${ }^{3}$ Dept. of Post Graduate Studies and Research in Physics and

Electronics, Rani Durgavati University, Jabalpur. Corresponding author E-mail: triptinanhoriya@gmail.co

\begin{abstract}
This paper presents the study of the structural and optical properties of copper doped cadmium sulphide nanoparticles by simple chemical route method. XRD pattern showed the hexagonal phase o\f CdS nanoparticles. Morphological analysis confirmed that the grain size increases on copper doping. Compositional analysis confirmed that $\mathrm{Cu}$ ions replace $\mathrm{Cd}$ ions either interstitial or substituitional in Cu: CdS nanoparticles. Increase in doping agent concentration leads to an increase in the optical transmission and increases the size of the particles as the band gap decreases.
\end{abstract}

Keywords: Nanocrystals, XRD, UV-Vis spectroscopy, FTIR.

\section{INTRODUCTION}

Cadmium Sulphide is a n-type semiconductor that has been extensively used in many applications using, including photo resistance sensors, field effect transistors, solar cells, photovoltaic, light emitting diodes, photocatalysis, photoluminiscence, infrared photodetector, environmental sensors and biological sensors[1]. Metal doping on CdS semiconductor results in the formation of the different type of nanostructure with novel properties and increases surface to volume ratio in nanoparticles enhances surface and interface effects resulting in novel phenomena[2]. CdS nanoparticles exhibit size dependent properties it has a band gap energy $\mathrm{E}_{\mathrm{g}}$ of $2.42 \mathrm{eV}$ at room temperature and pressure. Since CdS has wide band gap, it is used as window material 
for heterojunction solar cells to avoid the recombination of photogenerated carriers which improves the solar cell efficiency[3].

Copper impurity changes the type of CdS semiconductor from $n$ to $p$. The doping of copper changes the band gap energy of CdS and also improves its photoelectrical property[4]. Different methods have been used to dope the CdS films by $\mathrm{Cu}$, such as thermal annealing of $\mathrm{Cu} / \mathrm{CdS}$ bilayer[5]. The aim of the present work is to add a few percent of $\mathrm{Cu}$ and study the effect of $\mathrm{Cu}$ doping on the morphological and structural properties of the nanoparticles by Simple chemical route method.

\section{SYNTHESIS: MATERIALS AND METHODS}

Cadmium Sulphide nanoparticles were grown by Chemical Bath method using PVA as a capping agent. All the chemical reagents used were of analytical grade. For the undoped CdS nanoparticles, the solution of $\mathrm{CdSO}_{4}(0.5 \mathrm{M})$ was prepared by dissolving $\mathrm{CdSO}_{4}$ in DI water. The matrix solution was prepared by adding the $\mathrm{CdSO}_{4}$ solution to an aqueous solution $(2 \%)$ of PVA with constant stirring at room temperature to form a well dispersed PVA capped $\mathrm{Cd}^{2+}$ ion solution which is a transparent liquid indicating the complete dissolution of $\mathrm{CdSO}_{4}$. The $\mathrm{pH}$ of the solution was maintained at around 8 by slowly adding Ammonium Buffer Solution drop by drop to the above matrix solution to form the metallic complex. The thiourea as $\mathrm{S}^{2--}$ ion source was then added to the above metallic complex solution to form colloidal solution of CdS nanoparticles. For $\mathrm{Cu}$ doping, $\mathrm{CuSO}_{4}$ solution of $(0.005 \mathrm{M})$ was mixed with the host $\mathrm{CdSO}_{4}$ solution $(0.5 \mathrm{M})$ solution prior to deposition and similar steps were followed to have final CdS: Mn matrix solution.

\section{CHARACTERIZATION}

The prepared sample was taken for XRD study and UV studies. The structure of the sample is determined by X-ray diffraction measurements 
have been performed by using Xpert PANalytical instrument operating at $40 \mathrm{kV}$ and current of $30 \mathrm{~mA}$ with CuKa radiation. FTIR measurements are recorded using FTIR-8400s SHIMADZH (IR solution). UV-Visible spectrum of the nanoparticles is recorded using PERKIN ELMER UV-Vis spectrophotometer.

\section{RESULTS AND DISCUSSION}

Optical absorption studies show that absorption edge shifts towards longer wavelengths by increasing loading of $\mathrm{Cu}$ doping indicating increase in crystal size. Large blue shift is observed in both $\mathrm{CdS}$ and $\mathrm{Cu}$ doped CdS samples with respect to bulk CdS. This indicates a clear quantum confinement in PVA capped CdS nanostructures. C.M. Janet et al also reported that, the size of CdS nanocrystal becomes smaller than the exciton radius a remarkable quantum size effect leads to a size dependent increase in the band gap and a blue shift in the absorption onset [6].

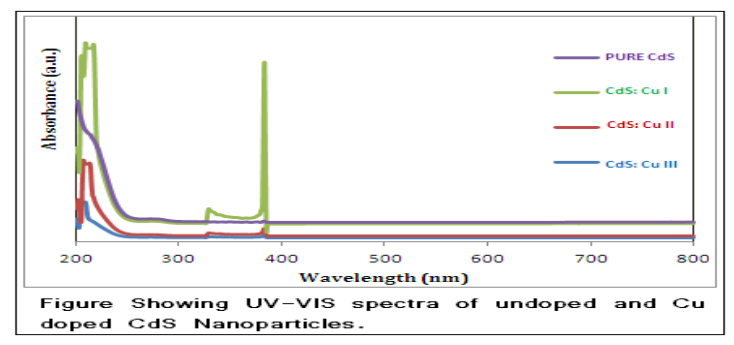

Fig 1. Absorption spectra of CdS:Cu nanoparticles

The structure of the prepared sample was investigated by $\mathrm{X}$-Ray Diffractometer technique. The XRD patterns of the sample is shown in fig. The particle size was calculated by using Debye-Scherrer formula.

$$
\mathrm{D}=\mathrm{k} \lambda / \beta \cos \theta
$$

Where, $\mathrm{k}$ is a dimensionless constant, $\lambda$ is the wavelength of $\mathrm{X}$-Ray used, $\beta=$ Full width at Half maxima (FWHM) of the diffraction peak and $\theta$ is the diffraction angle for the $(h, k, l)$ plane Bragg's angle. XRD shows hexagonal CdS nanoparticles with size in a few nanometers range. Significant peaks 
were obtained at $2 \theta$ angles $26^{\circ}, 29^{\circ}, 32^{\circ}, 36^{\circ}, 44^{\circ}, 51.5^{\circ}, 61.5^{\circ}$ corresponds to the reflections at (002), (101), (200),102), (110), (112), (202) planes.

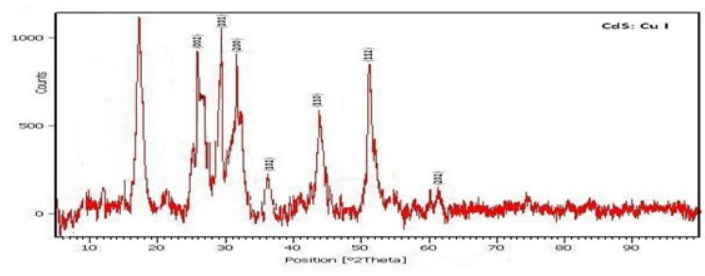

Figure 2(a): XRD spectra for CdS: Cu I sample.

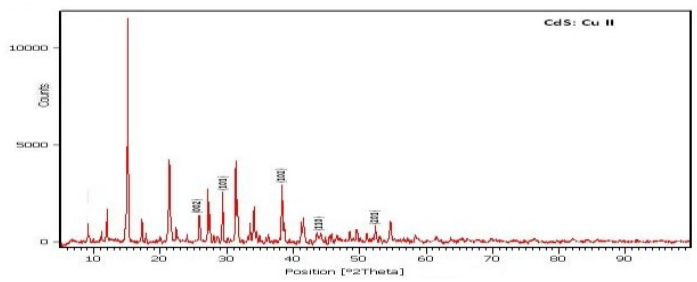

Figure 2(b): XRD spectra for CdS: Cu II sample.

In FTIR spectrum, the peak at $3431 \mathrm{~cm}^{-1}$ is assigned to $\mathrm{O}-\mathrm{H}$ stretching of absorbed water on the surface of the sample and the peak at $1438 \mathrm{~cm}^{-1}$ is attributed to bending vibrations of Poly Vinyl Alcohol used in the process. The C-O stretching vibration of absorbed PVA molecule gives its intense peak at $1025 \mathrm{~cm}^{-1}$. In addition to surface coverage of CdS by PVA, presence of trace amount of template ligand namely PVA is also evident, its ring C-H vibration occurs at about $3074 \mathrm{~cm}^{-1}$ which is a very weak peak. The FT-IR spectra for CdCuS sample is shown in figure 3(a) and figure $3(\mathrm{~b})$. 


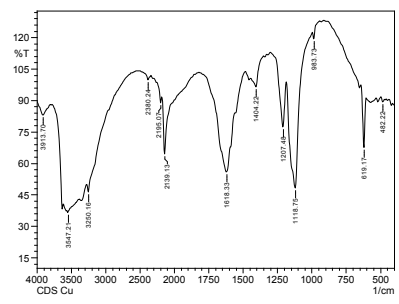

Figure 3(a): FTIR spectra for CdS: Cu I sample.

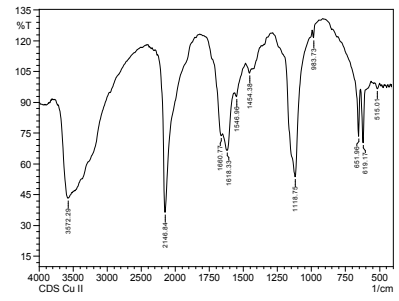

Figure 3(b): FTIR spectra for CdS: Cu II sample.

\section{CONCLUSIONS}

A systematic study of structural and optical properties of $\mathrm{Cu}$ doped Cadmium Sulphide nanoparticles shows that CdS: $\mathrm{Cu}$ nanocrystalline samples were prepared successfully using simple chemical route method. FT-IR study revealed the formation of capping agent on the surface of the nanoparticles[6]. The structural characterization done through XRD revealed that the prepared samples are nano crystalline in nature[7]. The investigation revealed that $\mathrm{Cu}$ doped $\mathrm{CdS}$ nanoparticles have suitable structural parameters in view of the solar cell applications[8]. 


\section{REFERENCES}

Charles Kittel, (1995) Introduction to Solid State Physics, 7.

S. A. Al Kuhaimi, (1998) Sol. Energy Mater. Sol. Cells 52, 69 [DOI:10.1016/S0927-0248(97)002729].

B.Srinivasa Rao, B.Rajesh Kumar, V.Rajagopal Reddy, T.Subba Rao, (March 2011) Preparation and Characterization of CdS Nanoparticles by Chemical co-precipitation technique, Chalcogenide Letters, Vol. 8, No. 3, p.177 - 185.

Be Xuan Hop, Ha Van Trinh, Khuc Quang Dat, Phung Quoc Bao , (2008) Growth of CdS thin films by chemical bath deposition technique, VNU Journal of Science, Mathematics - Physics 24 119-123.

S.Salimian and S.Farjami Shayesteh, (2010) Luminescence Properties of CdS Nanoparticles under Various Synthesis Conditions, Vol. 118, pp.4.

C M Janet and R P Viswanath, (2006) Large Scale synthesis of CdS nanorods and its utilization in photo catalytic H2 production technology, Nanotechnology 17, 5271-5277.

S.Linsi, B.Kavitha, M.Dhanam, and B.Maheshwari, (2010) Analysis of Cu: CdS Thin Films of Three different copper concentration, World Applied Sciences Journal 10(2): 201 - 213, 2010, ISSN1818-4952 CIDOSI Publications.

V.I. Klimov, (2006) J. Phys. Chem; B 110, 16827. 